\title{
Le réseautage : Une activité de santé publique par excellence
}

\author{
Michel Deilgat ${ }^{1}$, Patricia Huston ${ }^{1}$
}

Il est surprenant de constater que relativement peu de choses ont été écrites au sujet du réseautage, alors qu'il s'agit d'un élément indispensable à la planification, à la coordination et à l'exécution des activités de santé publique. Cela peut s'expliquer en partie par le fait que le réseautage est décrit de multiples façons : coordination de la mobilisation des intervenants, travail pluridisciplinaire et approche intersectorielle. Cela pourrait aussi être dû au fait que les réseaux sont tellement inscrits dans les gènes de la santé publique que l'on prend rarement le recul nécessaire pour en apprécier leur valeur... un peu comme un poisson qui se contente de présumer l'existence de l'eau.

Un réseau de santé publique est un groupe de personnes qui représentent des organisations, des intérêts ou des ordres de gouvernement et qui travaillent de concert dans le but d'améliorer la santé publique. Les réseaux facilitent l'interaction sociale, le partage des connaissances et la participation à d'autres activités en lien avec un but commun dans un domaine précis de connaissances et de pratique (1). À bien y réfléchir, il est difficile d'imaginer la santé publique sans les réseaux.

Cette édition de février 2020 du Relevé des maladies transmissibles au Canada (RMTC) a pour thème ces réseaux de santé publique. Découvrez un « réseau de réseaux »: les centres de collaboration nationale du Canada (CCN) (2). Les CCN représentent six centres d'expertise composés d'équipes pluridisciplinaires dont les membres agissent en tant que " courtiers " de connaissances afin de cerner les lacunes et de synthétiser, de partager et d'utiliser les connaissances en vue de promouvoir la prise de décisions éclairées par des données probantes en matière de santé publique. Pour ce faire, ils emploient une stratégie, caractéristique de la santé publique, qui consiste à réseauter à tous les niveaux du système de santé publique canadien, dans les autres disciplines et à l'échelle internationale pour faciliter l'utilisation des connaissances dans les politiques et la pratique. Le Comité consultatif national de l'immunisation (CCNI) offre un autre excellent exemple du pouvoir du réseautage. Le CCNI est un comité pluridisciplinaire dont les membres possèdent l'un des savoirs les plus complets du pays en matière de vaccins. Ces membres résument les données probantes et formulent des recommandations de santé publique et de soins cliniques. Créé en 1964, le CCNI constitue, depuis près de 60 ans, une source fiable de renseignements sur l'immunisation pour le Canada $(3,4)$. Découvrez dans cette édition ses travaux les plus récents, ainsi qu'un résumé de sa dernière prise de position concernant l'utilisation de Trumenba ${ }^{\mathrm{MC}}$ pour la prévention du méningocoque de sérogroupe $B(5)$. Vous trouverez également à la fin de cette édition plusieurs actualités sur les maladies infectieuses, dont les résultats d'une étude attendue depuis longtemps sur l'efficacité du réseautage et des partenariats destinés à répondre aux priorités de santé publique.

Le réseautage comme stratégie de santé publique mérite plus de reconnaissance et doit être étudié de manière plus approfondie. Bien que le réseautage fasse partie intégrante des activités de santé publique, paradoxalement, peu d'études ont cherché à en évaluer son efficacité. Nous devons bien entendu nous renseigner davantage sur ce qui peut fonctionner et ne pas fonctionner, ainsi que sur les pratiques exemplaires susceptibles de faciliter l'élaboration des futures stratégies de réseautage.

Citation proposée : Deilgat M, Huston P. Le réseautage : une activité de santé publique par excellence. Relevé des maladies transmissibles au Canada 2020;46(2/3):33-4. https://doi.org/10.14745/ccdr.v46i23a01f 


\section{Références}

1. Robeson P. Le réseautage en santé publique : analyse de la valeur des réseaux pour les Centres de collaboration nationale en santé publique. Centre de collaboration nationale des méthodes et outils. Hamilton (ON): CCNSP ; 2009 (Accédé 2020-01-02). https://www.nccmt.ca/ pubs/NetworkingPaperAprO9EN_WEB.pdf

2. Dubois A, Lévesque M. Les Centres de collaboration nationale du Canada : Faciliter la prise de décisions informées par des données probantes en matière de santé publique. Relevé des maladies transmissibles au Canada, 2020;46(2/3):35-9. DOI

3. Gemmill I. Comité consultatif national de l'immunisation (CCNI) : Célébration de 50 ans de service. Relevé des maladies transmissible au Canada 2014 Oct;40(17):374-6. $\mathrm{DOI}$
4. Desai S, Lsmail SJ, Lerch R, Warshawsky BF, Gemmill I. Canada's National Advisory Committee on Immunization: Celebrating 50 years. Can J Infect Dis Med Microbiol 2015 May-Jun;26(3):126-8. DOI PubMed

5. Harrison R, Stirling R, Baclic $O$, Vaudry W. Résumé de la déclaration du CCNI sur l'utilisation du vaccin bivalent dirigé contre la protéine de liaison au facteur $\mathrm{H}$ (MenB-fHBP) pour la prévention de l'infection à méningocoque du sérogroupe B. Relevé des maladies transmissible au Canada 2020;46(2/3):40-4. DOI 